\title{
Aprendizado de adultos
}

\author{
Adult learning
}

Alessandro Giraldes Iglesias'; Antonio Pazin-Filho²

\begin{abstract}
RESUMO
Gradativamente, o aprendizado de adultos tem se diferenciado da pedagogia. Isso começou com a psicologia cognitiva e atualmente já se dispõe de evidências de neuroimagem que justifiquem essas diferenças e permitam o melhor conhecimento do processo de aprendizagem e memória para embasar o desenvolvimento de novas técnicas de ensino.
\end{abstract}

Palavras-chave: Aprendizado de Adulto; Neurociência Cognitiva; Memória.

\section{Aprendizado de adultos}

As diversas demandas por resultados concretos do processo de ensino têm provocado inúmeras transformações, sendo uma das principais a aceitação de que o adulto tem necessidades específicas para o aprendizado. ${ }^{1,2}$ Num primeiro momento, houve a compreensão de que o adulto tem características diferentes das preconizadas pelas bases pedagógicas tradicionais e há cerca de uma década, a literatura testemunhou o nascimento da Andragogia e sua busca para se firmar como área específica. ${ }^{3,4}$ Posteriormente, houve o estreitamento de laços entre a psicologia cognitiva e a neurofisiologia para se compreender melhor o processo de aprendizado, não se importando tanto com a idade do indivíduo, mas com a compreensão dos diversos processos de aprendizado. Nasce então a Neurociência Cognitiva., 5,6,7

Apesar de promissora, ainda há diversas perguntas a serem respondidas para que se obtenha uma teoria unificada de aprendizagem e foge ao escopo desse artigo entrar em detalhes sobre todo o conhecimento que está sendo adquirido nessa área nascente. Nos limitaremos ao conhecimento necessário que se aplique ao construto teórico ("schema") que iremos utilizar ao longo desse texto. Mas é reconfortante saber que as diversas recomendações que se fizeram presentes no nascimento da Andragogia foram comprovadas e embasam recomendações de ensino baseado em evidências. ${ }^{8,9}$

Uma distinção que já deve ser realizada de início é entre a ciência de aprendizado e a ciência de
1. Médico formado na FMRP-USP; Especialista em Cardiologia; Pós-Graduando no Programa de Ensino em Saúde da FMRPUSP.

2. Professor Associado II da Divisão de Emergências Clínicas do Departamento de Clínica Médica da Faculdade de Medicina de Ribeirão Preto da Universidade de São Paulo. Coordenador do Laboratório de Simulação da FMRP-USP.
Correspondência: Prof. Dr. Antonio Pazin-Filho R. Bernardino de Campus, 1000 Ribeirão Preto - SP - CEP - 14010-030 Telefone - 16-3610-1110 apazin@fmrp.usp.br

Artigo recebido em 22/05/2014 Aprovado para publicação em 19/06/2014 
instrução. ${ }^{10}$ A primeira tem como objetivo compreender o processo de aprendizado, enquanto a segunda busca desenvolver técnicas de ensino que estejam embasadas no processo de aprendizado para facilitar a retenção de informação. Nesse artigo nos limitaremos a fornecer uma base conceitual mínima para a teoria de aprendizagem e memória, enquanto que as diversas metodologias de ensino serão motivo de vários outros artigos desse simpósio. Isso não quer dizer que não apontaremos eventuais ligações entre essas bases e os métodos que serão discutidos nesse simpósio.

Iniciaremos com uma descrição dos sistemas de memória classicamente descritos para fornecer um construto teórico ("schema") sobre o qual poderemos ilustrar diversos conceitos que sedimentam as recomendações de ensino baseado em evidências. Posteriormente, faremos uma síntese das características que devem ser levadas em consideração para o aprendizado do adulto que estão consolidadas e as correlacionaremos com a base metodológica de instrução.

\section{Memória e aprendizado}

Um dos paradigmas que foram quebrados ao longo de todo esse processo é de que o aluno é uma "tábula rasa", ou seja, desprovido de qualquer conhecimento sobre o assunto. Hoje sabemos que o aprendizado se inicia intra-útero e que é praticamente impossível que se lide com um aluno de qualquer idade que já não tenha algo a acrescentar sobre o assunto que está sendo ensinado, por menor que seja. Essa percepção alterou o conceito de aprendizado, sendo que uma definição mais apropriada seria o processo através do qual o aluno seleciona um conteúdo relevante de informação que lhe é oferecido, confronta-o com o conteúdo que já dispõe armazenado e realiza a síntese entre o novo e o antigo num conteúdo mais significativo. ${ }^{11}$

Frente a esse conceito, o estudo do aprendizado requer o conhecimento dos mecanismos de memória que dispomos, pois em última análise, queremos saber como podemos reter a informação que nos é oferecida.

A Figura 1 e a Tabela 1 apresentam um construto teórico ("schema") sobre os diversos processos de memorização. Através deles exploraremos aspectos importantes para o ensino de adultos. Ele tem base fundamentada em conceitos de psicologia cognitiva, mas a correlação com bases neuro-fisiológicas está se sedimentando (Tabela 1).

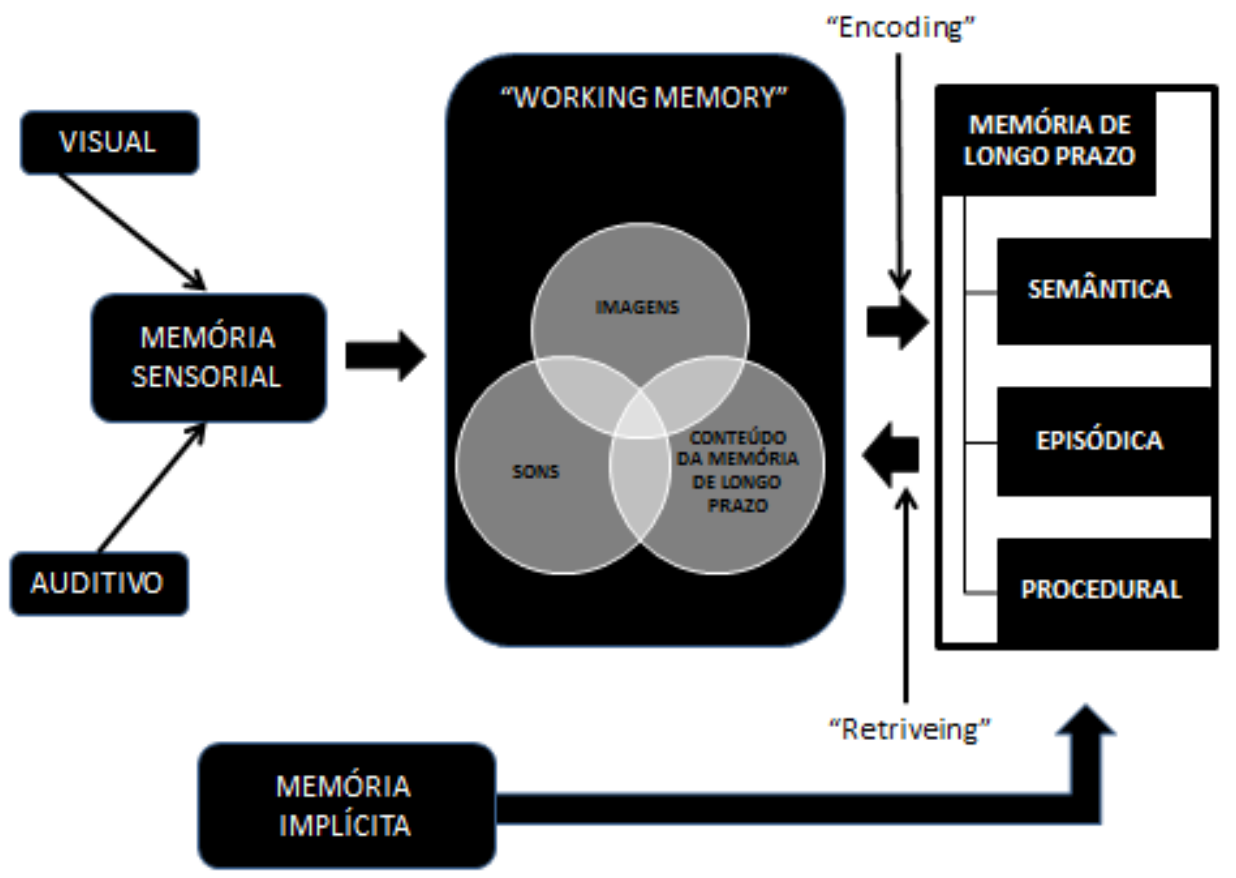

Figura 1: Componentes do Processo de Memória Humana. 
Tabela 1 - Conceitos importantes sobre os diversos tipos de processos de memorização.

\begin{tabular}{|c|c|c|}
\hline Tipo & Categoria & Base Anatômica \\
\hline Memória sensorial & $\begin{array}{l}\text { - Capacidade limitada, mas de maior amplitude que a "working } \\
\text { memory" } \\
\text { - Amplificada se diversos canais de comunicação estão sendo } \\
\text { utilizados simultaneamente }\end{array}$ & $\begin{array}{l}\text { Envolve todas as áreas senso- } \\
\text { riais do cérebro }\end{array}$ \\
\hline "Working Memory" & $\begin{array}{l}\text { - Tem capacidade limitada; } \\
\text { - Função de processar os diversos dados fornecidos pela me- } \\
\text { mória sensorial e cotejá-los com a informação contida na Me- } \\
\text { mória de Longo Prazo } \\
\text { - Capacidade de armazenamento pode ser amplificada por in- } \\
\text { formações concatenadas }\end{array}$ & $\begin{array}{l}\text { Envolve os lobos temporal e } \\
\text { occipital para origem da infor- } \\
\text { mação nova e o neocórtex para } \\
\text { obter a informação prévia para } \\
\text { integração }\end{array}$ \\
\hline \multirow[t]{4}{*}{ Memória de Longo } & $\begin{array}{l}\text { - Tem capacidade ilimitada } \\
\text { - Geralmente requer esforço considerável para retenção de in- } \\
\text { formação consciente } \\
\text { - Requer processamento via "working memory" ou através de } \\
\text { memória implícita }\end{array}$ & Neocórtex frontal \\
\hline & $\begin{array}{l}\text { - informação é armazendada dentro de um } \\
\text { contexto específico }\end{array}$ & $\begin{array}{l}\text { Neocórtex, mas há ativação de } \\
\text { outras áreas dependendo do } \\
\text { contexto em que foi gerada }\end{array}$ \\
\hline & $\begin{array}{l}\text { - informação é armazenada desprovida de } \\
\text { contexto, geralmente por exposições re- } \\
\text { petidas }\end{array}$ & Neocórtex frontal \\
\hline & $\begin{array}{l}\text { - informação pertinente à habilidades que } \\
\text { requerem informações detalhadas e que } \\
\text { nem sempre são conscientes }\end{array}$ & $\begin{array}{l}\text { Áreas relacionadas à procedi- } \\
\text { mentos repetitivos e inconsci- } \\
\text { entes }\end{array}$ \\
\hline Memória Implícita & $\begin{array}{l}\text { - Sistema relacionado ao aprendizado não-intencional } \\
\text { - Informação estocada relacionada ao contexto, mas de forma } \\
\text { inconsciente e não processada } \\
\text { - Estoca informações diferentes daquela absorvida pela rota prin- } \\
\text { cipal }\end{array}$ & Amigdala \\
\hline
\end{tabular}

\section{Memória sensorial}

Basicamente, os processos de memorização podem ser resumidos em dois tipos. Um primeiro pode ser chamado de rota principal, que envolve todo o processamento desde o estímulo até a estocagem de modo consciente. Já o segundo, diz respeito ao que aprendemos de modo inconsciente. Veremos uma des- crição de ambos considerando primeiro o aprendizado de um único conteúdo e depois discutiremos conteúdos paralelos.

A chamada rota principal se inicia com o estímulo que é oferecido. Esses estímulos são principalmente visuais e auditivos, ou seja, o aluno pode estar assistindo a um procedimento e cotejando as informações com o que está sendo explicado (estímulo 
auditivo). Esses estímulos são retidos inicialmente na memória sensorial, que pode reter uma quantidade considerável de informação, mas por tempo limitado e muito do que é capturado não é consciente. Nesse ponto inicial as imagens e sons obtidos são correlacionados e um conteúdo congruente é oferecido à "working memory".

Um ponto importante nessa etapa da memória sensorial é que ela pode ser amplificada se vários métodos de estímulo sensorial forem oferecidos. Evidências de neurofisiologia comprovam que quanto mais o aluno estiver envolvido na etapa de aquisição da informação, utilizando canais visuais e auditivos, concentrando sua atenção no que está sendo ensinado e mesmo atuando em modelos, a estruturação do conteúdo a ser retido é intensificada, já que várias áreas do cérebro são ativadas simultaneamente, e um produto mais trabalhado é oferecido à "working memory".

\section{"Working memory"}

A "working memory" (memória de trabalho) tem baixa capacidade de retenção de informação, mas apresenta duas funções principais para o processo de estocagem de informação. A primeira é sua capacidade de integrar a informação recebida e estabelecer um conteúdo sequencial que facilite o aprendizado. Nesse sentido, para aumentar sua capacidade de trabalho, a "working memory" cria pequenas "estórias" concatenadas do tipo "Era uma vez... aconteceu isso... e viveram felizes para sempre". O fato de se relacionar isso com estórias infantis é intencional, pois grande parte do aprendizado das crianças é feito dessa forma e parece ser uma condição também do adulto, justificando que os diversos métodos de ensino busquem essa estratégia para aumentar a retenção. Eles são a base do "schema" que será construído na memória de longo prazo. ${ }^{7}$

Deve ser ressaltado que, de toda a sequência dessa rota principal de aprendizado e memorização consciente, o ponto de restrição do sistema ("gargalo") é a "working memory" pela sua capacidade limitada de reter informação. Portanto, quaisquer métodos que amplifiquem a sua capacidade podem, teoricamente, implicar em maior retenção de conhecimento. ${ }^{.11}$

Um segundo ponto importante que ocorre na "working memory" é a integração do conteúdo novo com o que a pessoa já tem armazenado na memória de longo prazo. Para que possamos progredir nessa explicação, temos que introduzir o conceito de "schemas" (construtos teóricos).

O termo "schema" é geralmente utilizado para o conhecimento geral que a pessoa já dispõe armazenado. Eles estão associados ao processo de codificação/estocagem ("encoding") e recuperação ("retrieving") das informações na memória de longa permanência e estão imbuídos de informações cognitivas, procedurais, ambientais e culturais. Basicamente são mapas sobre um processo que estão estocados e serão atualizados com as novas informações. ${ }^{5}$

A Figura 2 ilustra dois "schemas", um prévio (A) e outro modificado (B) frente à intervenção educacional. Pode-se observar que no primeiro "schema" o aluno tem como base que frente à uma situação problema, ele dispõe de uma intervenção terapêutica a ser utilizada se não houver contra-indicações. Depois da intervenção, ele agora dispõe de dois tratamentos, sendo que o tratamento novo pode ser utilizado na presença de contra-indicações ao tratamento já conhecido. Essa intervenção ampliou o conhecimento do aluno que é resumido no "schema" B. Como houve modificação do "schema", pode-se dizer que houve aprendizado importante para a sua prática (chamado de significativo, não no sentido de magnitude, mas porque houve significância para que pudesse haver mudança de atitude).

Um ponto importante nesse conhecimento significativo é a motivação do aluno. Nesse ponto, como ele tem uma situação problema que está interessado em resolver, seu interesse em atualizar seu mapa conceitual é grande. Se o conhecimento transmitido já não tivesse o mesmo atrativo para resolver o problema, teríamos problemas com a retenção. Isso porque a congruência da informação antiga com a informação nova é um ponto crítico para a retenção. ${ }^{3}$

Outro ponto importante é não se entender essa etapa como linear. Na verdade, o aluno coteja o que conhece com o que está vendo e vai repetindo exaustivamente e de modo pró-ativo até se dar conta que o novo "schema" está atendendo suas necessidades. Essa percepção é muito importante para a retenção e é a base do "feed back" (retroalimentação). O melhor tipo de "feed back" é aquele em que o próprio aluno chega à síntese desejada, ou seja, um "insight". ${ }^{12}$ Mas outras modalidades de "feed back" podem ser utilizadas. O aluno não estará satisfeito enquanto esse novo "schema" for incongruente com o que está observando. Essa observação é importante, pois o aluno não 

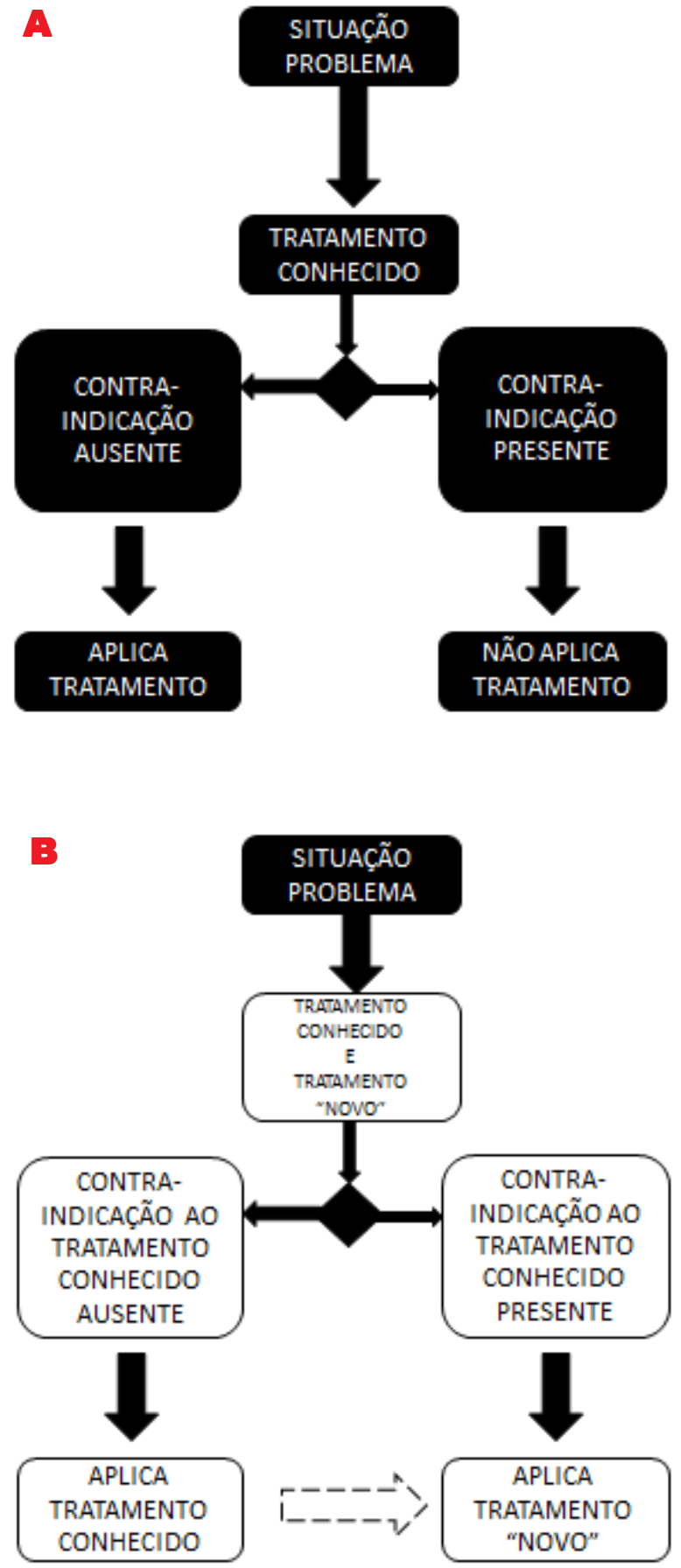

Figura 2 - Exemplo de "schema" anterior (A) e do novo "schema" (B) após a inserção da nova informação.

Obs: Em B estão destacados em branco as modificações feitas no "schema". O aluno agora tem uma opção de tratamento para a situação problema, ampliando suas opções terapêuticas. A seta tracejada ilustra uma pergunta decorrente da nova informação, que pode ou não ser reforçada pelo facilitador dependendo do nível de conhecimento ou do estágio do treinamento em que a pessoa esteja. Pode ser que essa seja uma situação que será explorada sequencialmente no currículo. conseguirá prosseguir no processo de aprendizado até que resolva essa pendência e os facilitadores devem estar atentos a esse processo.

A síntese entre o conhecimento disponibilizado e o conhecimento prévio, que resultou no "schema" B da Figura 2, passa agora a sofrer um processo de codificação/armazenamento ("encoding") na memória de longo prazo. Quando o conhecimento é recuperado para processamento, isso é chamado de "retrieving". Muitas vezes o construto teórico que temos sobre o armazenamento e recuperação da memória de longo prazo é como se estivéssemos utilizando um sistema de arquivo similar à uma gaveta. Quando aprendemos alguma coisa nova, apenas abrimos a gaveta e jogamos dentro. Na realidade, esse processo é dinâmico e não se trata apenas de acrescentar informação, mas de processar essa informação com significado, transformando-a. Há evidências de que esse processo não envolva apenas a simples síntese entre o novo e o antigo, mas que essa síntese seja influenciada pelas condições emocionais e ambientais em que está ocorrendo. Isso explica também o porque muitas vezes o que a pessoa recorda de um evento pode não ser verdade, mas sim a transformação do que ela observou à luz do que aprendeu. Provavelmente você já confrontou memórias com colegas em que ambos participaram do evento e notou que há discrepâncias nas memórias de ambos. Isso ilustra o processo e levanta questões sobre a validade do que se está observando e retendo. ${ }^{6}$

Um dos modos de contornar essa dificuldade é a exposição recorrente ao mesmo conteúdo, verificando-se se o "schema" continua contemplando as modificações que foram reforçadas. Ainda não há dados definitivos sobre o intervalo de re-exposição de um determinado conteúdo, mas parece que a repetição muito próxima pode não ser desejável. Mas de qualquer forma, isso embasa a necessidade de se elaborar um currículo longitudinal, com uma espiral virtuosa que permita a re-exposição frequente e o acréscimo de conteúdo, aprimorando o "schema". ${ }^{13}$

\section{Memória de longo prazo}

A memória de longo prazo tem três tipos de componentes. O primeiro é chamado de memória episódica, em que a informação do conhecimento é vinculada a um evento e em nível consciente. Isso é 
comum em situações de ensino em que o aluno vivenciou uma experiência significativa que resultou em aprendizado significativo.

O segundo tipo é a memória semântica, que implica na aquisição consciente de conteúdo cognitivo desprovido de um evento. Geralmente é fruto de exposições frequentes ao mesmo conteúdo mas em situações distintas. Como a associação com um processo sequencial facilita em muito a retenção, esse tipo de memória é mais difícil de se reter, pois geralmente está desprovido do contexto que facilita a estruturação de conteúdo sequencial.

Finalmente, há a memória procedural, relacionada ao aprendizado de habilidades, que pressupõem um conteúdo detalhado de conhecimento que nem sempre é consciente. Esse tipo de aprendizado é resultante de prática constante e uma vez adquirido, muitas vezes o aluno não consegue descrever porque está fazendo aquilo daquela forma. O melhor exemplo disso é aprender a dirigir. Provavelmente já está tão automático para você que se tiver que ensinar alguém, você terá que primeiro sentar no carro e decompor suas habilidades para ir demonstrando ao aluno. ${ }^{14}$

\section{Memória Implícita}

No segundo tipo de memorização, a memória implícita, o aprendizado ocorre de modo apenas parcialmente consciente, estando relacionado a eventos ou situações que nem sempre são reconhecidas como situações de aprendizado. Uma característica peculiar é que esse tipo de aprendizado não utiliza a "working memory", sendo armazenado diretamente na memória de longo prazo. Isso implica em armazenamento não processado e ligações com "schemas" anteriores de modo não programado. Conhecer esse tipo de aprendizado é importante pois confere importância à estruturação do ambiente a que o aluno é exposto durante o aprendizado e suas implicações para o currículo.
Deve-se ressaltar que a memória implícita é diferente do componente procedural da memória semântica, pois ela é não estruturada e se estabelece de modo inconsciente, sem controle do aluno ou do facilitador. Na memória procedural, o conhecimento é introduzido de modo consciente e à medida que vai sendo construído com repetição contínua, vai se tornando automático e semi-consciente.

Finalmente, devemos lembrar que tudo o que foi exposto se relacionou a um único conteúdo, quando na realidade, estamos aprendendo diversas coisas ao mesmo tempo. Ainda não se sabe se isso é benéfico ou não, se há diferença entre gerações (as novas gerações são chamadas de multi-tarefas, pois utilizam várias fontes de informação ao mesmo tempo) e como se fazer uso disso em técnicas de ensino. Além disso, há evidências de que o cérebro tenha capacidade para se adaptar para melhorar seu aprendizado através de neuroplasticidade, o que pode tornar o que foi exposto muito simplificado para conteúdos mais complexos. Finalmente, há estudos crescentes demonstrando uma variabilidade individual no aprendizado, que não tem sido levada em consideração, procurando-se alternativas do tipo "one size fits all". Ainda restam muitas perguntas a serem respondidas, mas acreditamos que o que foi exposto pode auxiliar em muito a estruturação do que deve ser ensinado, como discutiremos a seguir. ${ }^{4}$

\section{Características do aprendizado do adulto}

A Tabela 2 resume as características fundamentais desse processo de aprendizado e memória que devem ser bem compreendidos e utilizados no desenvolvimento de métodos e estratégias de ensino. Procurou-se ressaltar os conceitos, o porque eles são importantes, como se utilizar disso e exemplificar um método que se aproveite desse conceito. Sem dúvida há inúmeros outros métodos que se utilizem de um ou de vários dos pontos destacados. 
Tabela 2 - Características do aprendizado do adulto a serem consideradas na elaboração de métodos e estratégias de ensino.

\begin{tabular}{lll}
\hline Fato $\quad$ Implicação & Como se aproveitar disso & $\begin{array}{l}\text { Exemplo de técnica } \\
\text { de ensino }\end{array}$ \\
\hline
\end{tabular}

O aluno não é uma "tábula rasa"
Todos tem alguma informação em maior ou menor grau sobre o assunto.
Iniciar as atividades recordando o conhecimento que se deseja que o aluno já tenha
Ensino Baseado em Problema

\begin{abstract}
A base do armazenamento ("encoding") são "schemas". Deve haver congruência entre as informações novas e as antigas para que se promova a síntese
\end{abstract}

O "schema” é uma síntese do que a pessoa aprendeu e é pictográfica.
Desenvolver técnicas de ensino na qual se sintetize o que pessoa já sabe e utilizar as intervenções para modificar as figuras produzidas.
Fluxogramas

Simulação

Para habilidades, isso pode ser adquirido com simuladores em que se possa praticar o que se deseja reter

\begin{abstract}
Pró-atividade
$\mathrm{O}$ adulto tem necessidade de se envolver ativamente no processo de aprendizagem

dade de informação quando que está sendo ensinado é congruente com o que ele necessita aprender.Algumas formas de motivação envolvem o reconhecimento por pares
\end{abstract}

Especificar claramente o que se pretende com a atividade. Estabelecimento do contrato de ensino
Ensino baseado em Equipes
Re-exposição
A re-exposição do conteúdo é necessária para sedimentação de um conceito válido, pois o processo de memorização pode sofrer influências que comprometam a retenção de algo válido
Ainda não se tem certeza de qual o período mínimo de reexposição. Se for muito frequente pode inclusive ser danoso. Mas deve-se sempre recuperar o que a pessoa já sabe antes de expô-la a novos conhecimentos
Desenvolvimentos de eixos longitudinais de ensino com espirais virtuosas de acréscimo de conhecimento
"Feed back" - o aluno precisa de retro-alimentação para saber se o que está fazendo está certo ou não
Há vários modos de se obter isso, mas a melhor maneira é permitir que o próprio aluno chegue à conclusão de que aprendeu o conceito
Para que isso ocorra, tem que haver espaço para o aluno praticar inúmeras vezes para que possa testar se seu novo "schema" é eficaz ou não. Enquanto isso não for atingido, o aluno não conseguirá ir para frente no processo de aprendizado.
Simulação 


\section{ABSTRACT}

Gradually, adult learning has been differentiated pedagogy. This began with the cognitive psychology and currently already have neuroimaging evidence to justify these differences and allow a better understanding of the learning and memory process to support the development of new teaching techniques.

Key-words: Adult Learning; Cognitive Neuroscience; Memory.

\section{Referências Bibliográficas}

1. Collins J. Education techniques for lifelong learning - principles of adult learning. Radiographics. 2004;24:1483-9.

2. Ansari D, Coch D. Bridges over troubled waters: education and cognitive neuroscience. Trends Cogn. Sci. [Internet]. 2006 May [cited 2013 Oct 31];10:146-51. Available from: http://www.ncbi.nlm.nih.gov/pubmed/16530462

3. Horii CV. Teaching insights from adult learning theory. J. Vet. Med. Educ. [Internet]. 2007 Jan;34:369-76. Available from: http://www.ncbi.nlm.nih.gov/pubmed/18287460

4. Goldman S. The Educational Kanban: promoting effective self-directed adult learning in medical education. Acad. Med. [Internet]. 2009 Jul;84:927-34. Available from: http:// www.ncbi.nlm.nih.gov/pubmed/19550191

5. Patel VL, Yoskowitz N a, Arocha JF, Shortliffe EH. Cognitive and learning sciences in biomedical and health instructional design: A review with lessons for biomedical informatics education. J. Biomed. Inform. [Internet]. Elsevier Inc.; 2009 Mar [cited 2013 Oct 31];42:176-97. Available from: http:// www.ncbi.nlm.nih.gov/pubmed/19135173

6. Carew TJ, Magsamen SH. Neuroscience and education: an ideal partnership for producing evidence-based solutions to Guide 21(st) Century Learning. Neuron [Internet]. Elsevier Inc.; 2010 Sep 9 [cited 2014 Jan 29];67:685-8. Available from: http://www.ncbi.nlm.nih.gov/pubmed/20826300

7. Hruby GG. Three requirements for justifying an educational neuroscience. Br. J. Educ. Psychol. [Internet]. 2012 Mar [cited 2013 Oct 31];82(Pt 1):1-23. Available from: http:// www.ncbi.nlm.nih.gov/pubmed/22429055
8. Pazin-Filho A. Características do aprendizado do adulto. Medicina (Ribeirão Preto). 2007;40:7-16.

9. Neves FF, Pazin-Filho A. Raciocínio clínico na sala de urgência. Medicina (Ribeirão Preto).2008;41:1-8.

10. Mayer RE. Applying the science of learning to medical education. Med. Educ. [Internet]. 2010 Jul [cited 2013 Oct 31];44(6):543-9. Available from: http://www.ncbi.nlm.nih.gov/ pubmed/20604850

11. Ruiter DJ, van Kesteren MTR, Fernandez G. How to achieve synergy between medical education and cognitive neuroscience? An exercise on prior knowledge in understanding. Adv. Health Sci. Educ. Theory Pract. [Internet]. 2012 May [cited 2013 Oct 31];17(2):225-40. Available from: http:// www.pubmedcentral.nih.gov/articlerender.fcgi?artid=3319883 \&tool=pmcentrez\&rendertype=abstract

12. Pazin-filho A, Scarpelini S. Simulação: definição. Medicina (Ribeirão Preto).2007;40:162-6.

13. Cutting MF, Saks NS. Twelve tips for utilizing principles of learning to support medical education. Med. Teach. [Internet]. 2012 Jan [cited 2013 Oct 31];34:20-4. Available from: http:// www.ncbi.nlm.nih.gov/pubmed/21777127

14. Friedlander MJ, Andrews L, Armstrong EG, Aschenbrenner C, Kass JS, Ogden P, et al. What Can Medical Education Learn From the Neurobiology of Learning/? 2011;86:415-20. 